\title{
Stability of Compressible Boundary Layers Over a Smooth Backward-Facing Step
}

\author{
by \\ S. A. Ragab, A. H. Nayfeh, and R. C. Krishna \\ Department of Engineering Science and Mechanics \\ Virginia Polytechnic Institute and State University \\ Blacksburg, Virginia 24061
}

\begin{abstract}
An investigation is conducted into the determination of the credibility of interacting boundary layers in predicting compressible subsonic flows over smooth surface imperfections. The case of smooth backward-facing steps is considered. The predicted mean flows are compared with those obtained using a Navier-Stokes solver. Moreover, the linear 2-D compressible stability characteristics of both mean flows are compared. The results show that the interacting boundary-layer formulation produces accurate mean flows that yield accurate linear stability characteristics, such as growth rates and amplification factors.
\end{abstract}

PAC Numbers: $47.20 \mathrm{Ft}, 47.20 \mathrm{Ky}$

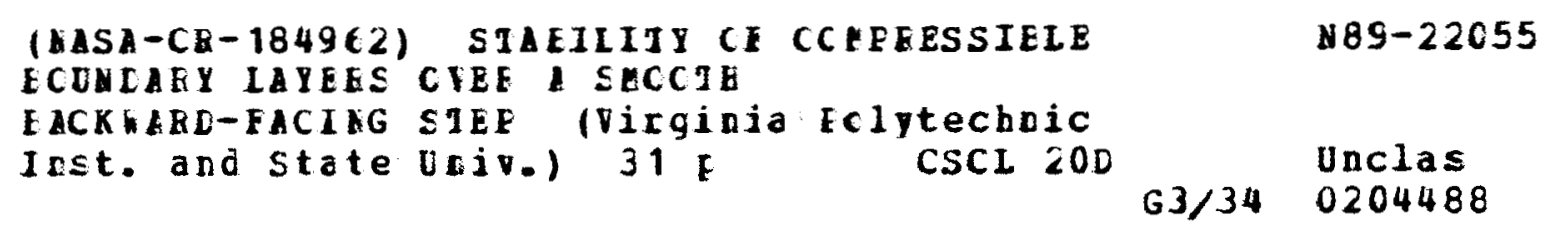




\section{Introduction}

The boundary layers over natural laminar flow components in the presence of surface imperfections (e.g., waviness and steps) must accurately be computed so that the effect of these imperfections on the stability and transition can be evaluated. Moreover, the magnitudes of the imperfections under consideration are such that strong viscid-inviscid interaction and small separation bubbles are unavoidable. Definitely, solutions to the full Navier-Stokes equations can accurately predict such flowfields provided that the grid is fine enough so that important flow structures are not smeared by the truncation errors or artificial dissipation. However, the number of flow cases that needs to be investigated is very large, and hence solving the full Navier-Stokes equations is a very expensive task. A more economical alternative is to solve the interacting boundary-layers equations.

The purpose of the present paper is to investigate the accuracy of the compressible interacting boundary-layer computations and to establish their credibility in accurately predicting flows over surface imperfections. Specifically, we consider the flow past a flat plate having a smooth backward-facing step. Our approach is to compare the results of interacting boundary-layer computations with solutions to the Navier-Stokes equations. Comparisons are made for the mean-flow profiles as well as the stability characteristics, such as, the growth rates and amplification factors of linear stability waves.

\section{Mean Flow}

\section{A. Navier-Stokes Solver}

The thin-layer compressible Navier-Stokes equations were solved using the well known computer code "ARC2D" which was developed at NASA Ames; the version that we acquired from NASA Ames is designated 1.5 GAMMA, $7 / 2 / 85$. The code incorporates different methods of solution, all of which are implicit in time, and uses second-order central differences in space. We 
selected the method of solution in which the diagonal form of the equations is used'. Mixed second- and fourth-order dissipation terms were added explicitly and implicitly, and the resulting pentadiagonal system of equations were solved directly.

Velocities are normalized using the freestream velocity $U_{\infty}^{*}$, lengths are normalized using the distance $L^{*}$ from the leading edge of the plate to the center of the step, and the temperature, viscosity, and thermal conductivity coefficients are normalized using their freestream values $T_{\infty}, \mu_{\infty}^{*}$, and $\kappa_{\infty}^{*}$, respectively. Sheared Cartesian grids are used for all the cases analyzed in this paper. An example is shown in Fig. 1 for a smooth backward-facing step. The equation of the step is

$$
y=\frac{1}{2} h(1+e r f \zeta)
$$

where

$$
\zeta=R e^{-3 / 8} \lambda^{5 / 4}(x-1)
$$

$\mathrm{Re}$ is the Reynolds number based on the distance from the leading edge to the step center $\left(x=x^{*} / L^{*}=1\right)$, and $\lambda=0.332057$. The step specified by Eq. (1) was originally employed by Smith and Merkin ${ }^{2}$ who analyzed the incompressible mean flow using triple-deck theory. The numerical values used in Fig. 1 are $R e=10^{\circ}$ and $h=-0.003$. We note that the $y$-coordinates in Fig. 1 are magnified by a factor of 20 relative to the $x$-coordinates.

The inflow boundary of the computational domain is located at $x \simeq-0.06$ (i.e., the plate leading edge $x=0$ is included in the domain) and the outflow boundary is located at $x=2.0$. The top boundary is placed at $y \simeq 0.4$. More details about the grid are given in Section IV.

\section{B. Interacting Boundary Layers}

We developed a code for solving the compressible interacting boundary-layer equations. The numerical method is an extension of Veldman's method ${ }^{3}$ to compressible flows. The salient feature of the method 
is the simultaneous solution of the boundary-layer equations and the inviscid flow, which is given by the small disturbance theory of compressible potential flow. A similar treatment was presented by Davis ${ }^{4}$ for subsonic and supersonic flows over parabolic humps. For more details about our method we refer the reader to Ref. 5.

The governing equations are the compressible boundary-layer equations. In terms of a combination of the Levy-Lees variables and the Prandtl transposition theorem, the problem is given by

$$
\begin{gathered}
2 \xi F F_{\xi}+V F_{\eta}-\frac{\partial}{\partial \eta}\left(\theta \frac{\partial F}{\partial \eta}\right)+\beta_{0}\left(F^{2}-Q\right)=0 \\
2 \xi F_{\xi}+V_{\eta}+F=0 \\
2 \xi F Q_{\xi}+V Q_{\eta}-\frac{\partial}{\partial \eta}\left(\frac{\theta}{P r} \frac{\partial Q}{\partial \eta}\right)-(\gamma-1) M_{\infty}^{2} \frac{U_{e}^{2}}{T_{e}} \theta F_{\eta}^{2}=0
\end{gathered}
$$

subject to the boundary conditions

$$
\begin{gathered}
F=0, V=0, Q_{\eta}=0 \text { at } \eta=0 \\
F \rightarrow 1 \text { and } Q \rightarrow 1 \text { as } \eta \rightarrow \infty \\
F=F\left(\xi_{0}, \eta\right) \text { and } Q=Q\left(\xi_{0}, \eta\right) \text { at } \xi=\xi_{0}
\end{gathered}
$$

where

$$
\begin{gathered}
\xi(x)=\int_{0}^{x} \rho_{e} \mu_{e} U_{e} d x \text { and } \eta(x, z)=\frac{U_{e} \sqrt{R e}}{\sqrt{2 \xi}} \int_{0}^{z} \rho d x \\
F=\frac{u}{U_{e}}, Q=\frac{T}{T_{e}}, z=y-f[\zeta(x)] \\
V(\xi, \eta)=\frac{\sqrt{2 \xi}}{\rho_{e} U_{e} \mu_{e}}\left[\rho\left(v-u \frac{d f}{d x}\right) \sqrt{R e}+\eta_{x} \sqrt{2 \xi} F\right]
\end{gathered}
$$




$$
\operatorname{Pr}=\frac{\dot{\mu}_{\infty}^{*} C_{p}^{*}}{\dot{\kappa_{\infty}^{*}}}, R e=\frac{\dot{U}_{\infty}^{*} L^{*} \dot{\rho}_{\infty}^{*}}{\dot{\mu_{\infty}}}, y=\frac{\dot{C}_{p}^{*}}{C_{v}^{*}}, \theta=\frac{\rho \mu}{\rho_{\mathrm{e}} \mu_{e}}, \beta_{0}=\frac{2 \xi}{U_{e}} \frac{d U_{e}}{d \xi}
$$

and $C_{p}^{*}$ and $C_{v}^{*}$ are the coefficients of the specific heat of the gas at constant pressure and volume, respectively. Here $\rho_{e}, \mu_{e}$, and $U_{e}$ are the edge density, viscosity coefficient, and velocity, respectively. The displacement thickness is given by

$$
\delta=\frac{1}{\sqrt{R e}} \frac{\sqrt{2 \xi}}{\rho_{e} U_{e}} \int_{0}^{\infty}(Q-F) d \eta
$$

The interaction law relates the edge velocity $U_{e}$ to the displacement surface. Using thin airfoil theory, we obtain

$$
U_{e}=\bar{U}_{e}+\frac{1}{\beta \pi} \int_{L . E .}^{\infty} \frac{U_{e} \delta}{x-t} \frac{d\left(\ln \rho_{e}\right)}{d t}+\frac{1}{\beta \pi} \int_{L . E .}^{\infty} \frac{1}{x-t} \frac{d\left(U_{e} \delta\right)}{d t} d t
$$

where

$$
\beta=\sqrt{1-M_{\infty}^{2}}
$$

and $\bar{U}_{e}$ is the inviscid surface velocity in the absence of the boundary layer, which is also determined using thin airfoil theory. After some manipulations, the interaction law can be rewritten as

$$
U_{e}=1+\frac{1}{\beta \pi} \int_{L . E .}^{\infty} \frac{1}{x-t} \frac{d \chi}{d t}+\frac{1}{\beta \pi} \int_{L . E .}^{\infty} \frac{U_{e} \delta}{x-t} \frac{d\left(\ln \rho_{e}\right)}{d t} d t
$$

where

$$
\chi=f+U_{e} \delta
$$

and the principal values of the Cauchy integrals in Eqs. (14) and (16) are assumed. Equations (3)-(8), (13), and (16) are solved simultaneously following the procedure of Veldman ${ }^{3}$. Veldman integrated Eq. (16) by parts to obtain a second derivative for $\chi$ and expressed $U_{\mathrm{e}}$ as a linear combination of the values of $\delta$ at the nodes. However, we follow Davis and Werle ${ }^{6}$ and Nayfeh et al ${ }^{5}$ and 
perform the integration by parts to eliminate the derivative of $\chi$ and assume $\chi$ to vary linearly over the differencing intervals, resulting in a second-order accurate scheme. The finite-difference scheme used employed a three-point upwind differencing formula for the $\xi$ derivatives and a central differencing for the $\eta$ derivatives. Upwinding the $\xi$ derivatives stabilizes the numerical method in regions of reversed flow (i.e., $F<0$ ). The system of equations is solved by a scheme similar to that employed by Nayfeh et $\left.a\right|^{5}$. The distribution of the mesh points on the steps was chosen in accordance with the triple-deck scaling. The lower deck was resolved using a mesh with a variable step size in the $\eta$ direction so that a larger number of points could be employed near the wall than in the rest of the boundary layer.

\section{Stability Formulation}

We consider the linear quasiparallel two-dimensional stability of two-dimensional compressible flows over smooth backward-facing steps. The quasiparallel assumption can be justified a posteriori ${ }^{5}$. The calculated wavelengths are the order of the boundary-layer thickness. We superpose a small time dependent disturbance on each mean-flow, thermodynamic, and transport quantity. Thus, we let

$$
\hat{q}(x, y, t)=q_{m}(y)+q(x, y, t)
$$

where $q_{m}(y)$ is a two-dimensional basic-state quantity and $q(x, y, t)$ is a two-dimensional unsteady disturbance quantity. Here, $\hat{q}$ stands for the velocity components ( $u$ and $v$ ), temperature $T$, pressure $p$, density $\rho$, and viscosity coefficient $\mu$. Substituting Eq. (18) into the Navier-Stokes equations, subtracting the basic-state quantities, and linearizing the resulting equations in the $q^{\prime} s$, we obtain

$$
\frac{\partial \rho}{\partial t}+\rho_{m} \frac{\partial u}{\partial x}+u_{m} \frac{\partial \rho}{\partial x}+\frac{\partial}{\partial y}\left(\rho_{m} v\right)=0
$$




$$
\begin{gathered}
\rho_{m}\left(\frac{\partial u}{\partial t}+u_{m} \frac{\partial u}{\partial x}+v \frac{d u_{m}}{d y}\right)=-\frac{\partial p}{\partial x}+\frac{1}{R}\left\{\mu _ { m } \frac { \partial } { \partial x } \left(r \frac{\partial u}{\partial x}\right.\right. \\
\left.\left.+m \frac{\partial v}{\partial y}\right)+\frac{\partial}{\partial y}\left[\mu_{m}\left(\frac{\partial u}{\partial y}+\frac{\partial v}{\partial x}\right)+\mu \frac{d u_{m}}{d y}\right]\right\} \\
\rho_{m}\left(\frac{\partial v}{\partial t}+u_{m} \frac{\partial v}{\partial x}\right)=-\frac{\partial p}{\partial y}+\frac{1}{R}\left\{\mu_{m} \frac{\partial}{\partial x}\left(\frac{\partial u}{\partial y}+\frac{\partial v}{\partial x}\right)\right. \\
\left.+\frac{\partial \mu}{\partial x} \frac{d u_{m}}{d y}+\frac{\partial}{\partial y}\left[\mu_{m}\left(m \frac{\partial u}{\partial x}+r \frac{\partial v}{\partial y}\right)\right]\right\} \\
\rho_{m}\left[\frac{\partial T}{\partial t}+u_{m} \frac{\partial T}{\partial x}+v \frac{d T_{m}}{d y}\right]=(y-1) M_{\infty}^{2}\left[\frac{\partial p}{\partial t}+u_{m} \frac{\partial p}{\partial x}+\frac{\mu}{R}\left(\frac{d u_{m}}{d y}\right)^{2}\right. \\
\left.+\frac{2 \mu_{m}}{R} \frac{d u_{m}}{d y}\left(\frac{\partial u}{\partial y}+\frac{\partial v}{\partial x}\right)\right]+\frac{1}{R \rho_{r}}\left\{\frac { \partial } { \partial y } \left(\mu_{m} \frac{\partial T}{\partial y} \ldots\right.\right. \\
\left.\left.+\mu \frac{d T_{m}}{d y}\right)+\mu_{m} \frac{\partial^{2} T}{\partial x^{2}}\right\}
\end{gathered}
$$

where

$$
\begin{gathered}
R=\frac{U_{\infty}^{*} \delta_{r}^{*}}{v_{\infty}^{*}}, \delta_{r}^{*}=\left(\frac{v_{\infty}^{*} L^{*}}{U_{\infty}^{*}}\right)^{1 / 2} \\
m=\lambda_{m} / \mu_{m} \text { and } r=2+m
\end{gathered}
$$

The problem is completed by the specification of the boundary conditions; they are

$$
\begin{aligned}
& u=v=T=0 \text { at } y=0 \\
& u, v, T \rightarrow 0 \text { as } y \rightarrow \infty
\end{aligned}
$$

Next, we assume normal-mode solutions of the form 


$$
q(x, y, t)=\tilde{q}(y) \exp \left[i \int \alpha d x-i \omega t\right]
$$

where $\tilde{q}$ stands for the eigenfunctions of $u, v, p$, and T. Using Eq. (28) in Eqs. (19)-(23), (26), and (27) and dropping the tilde from $\tilde{q}$, we obtain the eigenvalue problem

$$
\begin{gathered}
D v=-i \alpha u+\frac{D T_{m}}{T_{m}} v+\frac{i \Omega p}{p_{m}}-\frac{i \Omega T}{T_{m}} \\
D^{2} u=\left(\frac{-i \rho_{m} \Omega R}{\mu_{m}}+r \alpha^{2}\right) u-\frac{\mu_{m}^{\prime} D T_{m}}{\mu_{m}} D u \\
+\left[\frac{\rho_{m} R D u_{m}}{\mu_{m}}-i \alpha \frac{\mu_{m}^{\prime} D T_{m}}{\mu_{m}}\right] v-i(1+m) \alpha D v \\
+\frac{i R \alpha}{\mu_{m}} p-\left[\frac{D u_{m}}{\mu_{m}} D\left(\mu_{m}^{\prime}\right)+\frac{D^{2} u_{m}}{\mu_{m}} \mu_{m}^{\prime}\right] T-\frac{\mu_{m}^{\prime}}{\mu_{m}} D u_{m} D T \\
\chi_{0} D p=-i \alpha\left(r \frac{D T_{m}}{T_{m}}+\frac{2 \mu_{m}{ }^{\prime} D T_{m}}{\mu_{m}}\right) u-i \alpha D u+\left(\frac{i R \Omega}{\mu_{m} T_{m}}-\alpha^{2}\right. \\
\left.+r \frac{D^{2} T_{m}}{T_{m}}+\frac{r \mu_{m}^{\prime}\left(D T_{m}\right)^{2}}{\mu_{m} T_{m}}\right) v+i \frac{r}{p_{m}}\left[\Omega\left(\frac{D T_{m}}{T_{m}}+\frac{\mu_{m}^{\prime}}{\mu_{m}} D T_{m}\right)(31)\right. \\
\left.-\alpha D u_{m}\right] p+\left[i \alpha D u_{m}\left(\frac{\mu_{m}^{\prime}}{\mu_{m}}+\frac{r}{T_{m}}\right)-\frac{i r \Omega \mu_{m}^{\prime}}{\mu_{m} T_{m}} D T_{m}\right] T-\frac{i r \Omega}{T_{m}} D T \\
D^{2} T=-2(\gamma-1) M_{\infty}^{2} P r D u_{m} D u+\left[R P r \frac{\rho_{m} D T_{m}}{\mu_{m}}-2 i(\gamma-1) \alpha M_{\infty}^{2} P r D u_{m}\right] v \\
+i(\gamma-1) M_{\infty}^{2} P r R \frac{\Omega}{\mu_{m}} p+\left[-i P r R \Omega \frac{\rho_{m}}{\mu_{m}}+\alpha^{2}-\frac{\mu_{m}^{\prime \prime}}{\mu_{m}}\left(D T_{m}\right)^{2}\right. \\
\left.-\frac{\mu_{m}^{\prime}}{\mu_{m}} D^{2} T_{m}-(\gamma-1) M_{\infty}^{2} P r \frac{\mu_{m}^{\prime}}{\mu_{m}}\left(D u_{m}\right)^{2}\right] T-2 \frac{\mu_{m}^{\prime} D T_{m}}{\mu_{m}} D T
\end{gathered}
$$

where 


$$
\begin{gathered}
\Omega=\omega-\alpha u_{m} \\
D=\frac{d}{d y}, \chi_{0}=\frac{R}{\mu_{m}}-i r \frac{\Omega}{p_{m}}, \text { and } p_{m}=\frac{\rho_{m} T_{m}}{\gamma M_{\infty}^{2}} \\
\mu_{m}^{\prime}=\frac{d \mu_{m}}{d T_{m}}, \mu_{m}^{\prime \prime}=\frac{d^{2} \mu_{m}}{d T_{m}^{2}}
\end{gathered}
$$

Equations (29)-(32) can be written as a system of six first-order equations of the form

$$
D \zeta=A \zeta
$$

where

$$
\zeta^{T}=\{u D u \vee p T D T\}^{T}
$$

The boundary conditions (33) and (34) can be written as

$$
\begin{gathered}
\zeta_{1}=\zeta_{3}=\zeta_{5}=0 \text { at } y=0 \\
\zeta_{n} \rightarrow 0 \text { as } y \rightarrow \infty
\end{gathered}
$$

The system of equations (38) subject to the boundary conditions (40) and (41) constitutes an eigenvalue problem. For a given Reynolds number, Mach number, and mean-flow profiles, we determine the dispersion relation

$$
g(\omega, \alpha)=0
$$

employing a numerical procedure. For the spatial stability problem considered here, we specify $\omega$ and an initial guess for the eigenvalue $\alpha$. We numerically integrate the system (38) from $y=y_{e}$ to $y=0$, where $y_{e}$ indicates a value of $y$ outside the boundary layer. In performing these calculations we used the computer code 'SUPORT' developed by Scott and Watts'. This code, which is based on the method of Gudonov, solves stiff two-point boundary-value problems. It uses the Runge-Kutta-Fehlburg scheme to integrate the equations and the Gram-Schmidt orthonormalization scheme to keep the solution vectors linearly independent. Normally, the first guess of the 
eigenvalue is incorrect and therefore one of the boundary conditions at $y=0$ is not satisfied. Then, a Newton-Raphson scheme is used to iterate on this unsatisfied boundary condition and obtain the eigenvalue to the desired accuracy.

Having found the growth rates $-\alpha_{i}$, we compute the $N$-factor as

$$
N=-\int_{R_{0}}^{R} 2 x_{j} d R
$$

where $R_{0}$ corresponds to the Reynolds number at the first neutral point. During the course of integration, if we find that $N$ becomes negative we set it equal to zero.

\section{Comparison Between Interacting Boundary Layers and Navier-Stokes Results}

In this section solutions for flows over smooth backward-facing steps obtained using the Navier-Stokes solver and IBL computations are compared. The Mach numbers considered for the comparison are $M=0.5$ and $M=0.8$. Comparisons are made for the mean flows as well as their stability characteristics, such as the growth rates and the $\mathrm{N}$-factors. To predict the flowfield and its stability accurately, we must choose a proper grid, which is fine enough to capture the important flow structures. The far field boundary also plays an important role in the determination of the solution. The boundary must be far enough to prevent contamination of the solution by reflection there, especially in the high subsonic Mach number case.

The grid used in the IBL calculations has a uniform step size of $\Delta x=0.005$ in the streamwise direction and a geometrically stretched grid in the $\eta$ direction. At the wall $\Delta \eta=0.05$ and the stretching factor is 1.05 . The edge of the boundary layer is taken at $\eta_{e}=8.0$. 
Four grids were used with the Navier-Stokes solver. In grid 1 (136x70), a uniform $\Delta x=0.005$ was used in the range $0.9 \leq x \leq 1.1$, where strong streamwise gradients are expected. For $x<0.9$ and $x>1.1, \Delta x$ is stretched geometrically at the rate of 1.05 provided that $\Delta x$ did not exceed 0.03 . If $\Delta x$ exceeded 0.03 , a uniform spacing $\Delta x=0.03$ was used. To cover the domain $-0.06 \leq x \leq 2.0,136$ points were used. The step size in the $y$ direction was geometrically stretched between the wall and the top boundary $y \approx 0.4$ with $\Delta y_{1}=1.5 \times 10^{-4}$, and the stretching factor was adjusted so that the number of points was 70 . In grid $2(136,99)$, the streamwise distribution of grid points was the same as in grid 1 , while the $y$ direction had 99 points with $\Delta y_{1}=0.3 \times 10^{-4}$. In grid 3 (166x120), the streamwise distribution in the interval $0.9 \leq x \leq 2.0$ was the same as that in grid 1 , but more points were added in the range $-0.06 \leq x \leq 0.9$ so that the total number of $x$-points was 166 . The purpose of this addition is to obtain a finer grid near the leading edge of the plate. The $y$ distribution was also refined so that 120 points were used between the wall and the top boundary $y \approx 0.4$ with $\Delta y_{1}=0.3 \times 10^{-4}$. Grid 4 was the finest grid used, where 176 points were used in the $x$-direction and 153 in the y-direction. In this grid, the streamwise distribution of grid points was the same as that in grid 1 except that we started with $\Delta x=0.003$ instead of 0.005 . The y grid had 153 points between the wall and the top boundary, which is placed now at $y \approx 0.8$ instead of the 0.4 , which was used for the first three grids, and $\Delta y_{1}=0.3 \times 10^{-4}$.

First we compare the mean-flow characteristics predicted by the IBL code with those obtained by the Navier-Stokes code. The friction-coefficient distributions on a backward-facing step with $\mathrm{h}=-0.003, \mathrm{Re}=10^{6}$, and $M_{\infty}=0.5$ are shown in Fig. 2. The Navier-Stokes results were obtained using grids 1,2 , and 3 . The corresponding pressure-coefficient distributions are shown in Fig. 3. We note that there is a great discrepancy between the IBL computations and the Navier-Stokes results when grid 1 (corse grid) is used. The discrepancy is obvious downstream of the separation point. That prompted grid refinements in the Navier-Stokes calculations. The results of the IBL computations are in good agreement with the results obtained using grids 2 and 3 almost everywhere except in the reattachment region. However, 
the excellent agreement between the results obtained using grids 2 and 3 , with grid 3 being the finer of the two, suggested that a further grid refinement may not result in an appreciable change in the reattachment region. And hence we proceeded with the stability analysis.

We fixed the frequency $\omega$ at a value of 80 , and solved the eigenvalue problem for the wavenumber $\alpha$. The mean flow was obtained by using the $1 B L$ code or by using the ARC2D code using grids 1,2 , and 3 . The growth rates $\left(-\alpha_{i}\right)$ are shown in Fig. 4 as a function of $R=\sqrt{R e_{x}}$ for the four sources of the mean flow. The corresponding $\mathrm{N}$-factors are shown in Fig. 5 . We note that the IBL results are far off from the results of grid 1 both for the growth rate and the $\mathrm{N}$-factor. A dramatic improvement was obtained when the finer grids 2 and 3 were used. Figure 5 shows that the IBL results are in better agreement with the results of grid 3 than with those of grid 2, especially upstream of the step. However, the IBL predicts $\mathrm{N}$-factors in the reattachment region that are lower than those predicted by using the two grids.

Next we decided to use a still finer grid in order to improve the agreement in the reattachment region. In grid 4 (176x153), we refined the grid notably in the $x$-direction, while the distribution in the $y$-direction was almost the same as in grid 3. In Fig. 6, we depict the friction coefficient distributions obtained by using the IBL code and the ARC2D code with grids $1,2,3$, and 4 . There is an obvious improvement in the agreement of the IBL and Navier-Stokes results in the reattachment region when grid 4 is used. In Fig. 7 we show the growth rates $\left(-\alpha_{i}\right)$ obtained by using the IBL mean flow and the ARC2D code with grid 4. The $\mathrm{N}$-factors are depicted in Fig. 8 , they show considerable improvement in the agreement between the IBL computations and the Navier-Stokes computations using grid 4.

A grid refinement study was also conducted at a Mach number of 0.8 . The mean flow and stability results show trends similar to those for the case of Mach number of 0.5 . The growth rates predicted by using the IBL and N-S mean flows are depicted in Fig. 9 for a backward-facing step of $h=-0.003$, $R e=10^{6}, M_{\infty}=0.8$, and $F=50 \times 10^{-6}$. The $N$-S mean flow is obtained by using grid 3. The corresponding $N$-factors are shown in Fig. 10. The agreement 
between the two methods of obtaining the mean flow is good. For more details, we refer the reader to Ref. 8 .

We conclude that for the purpose of the stability analysis of boundary layers over smooth surface imperfections, which might induce small separation bubbles, the interacting boundary-layer formulation is a viable aiternative to the Navier-Stokes equations.

\section{Effects of Mach Number}

Using interacting boundary-layer mean flow computations, we present in Figs. 11 and 12 the pressure coefficient and skin-friction coefficient, respectively, for a backward-facing step of $h=-0.003, \operatorname{Re}=10^{6}$, and $M_{\infty}=0.0,0.5$, and 0.8 . It is evident in Fig. 12 that increasing the Mach number increases the streamwise extent of the separation region. For each Mach number, we determined the most amplified frequency, that is, the frequency which results in the highest $\mathrm{N}$-factor. For $M_{\infty}=0.0,0.5$, and 0.8 , the corresponding frequencies are $(60,55$, and 50$) \times 10^{\cdots 6}$, respectively. The growth rates and $\mathrm{N}$-factors are shown in Figs. 13 and 14, respectively. As expected, the maximum growth rate is reduced by compressibility, but because of the increase in the separation region with increasing Mach number, the growth rate in the reattachment region is higher for high Mach numbers. Although the maximum $\mathrm{N}$-factor is reduced by increasing the Mach number, the $\mathrm{N}$-factor at the end of separation is practically equal for the three Mach numbers. The increase in the separation region offsets the stabilizing effects of compressibility.

\section{Acknowledgement}

This work was supported by the NASA Langley Research Center under Grant No. NAG-1-714 and the Office of Naval Research under Contract No. N00014-85-K-0011, NR 4325201. We thank Dr. Thomas H. Pulliam of NASA Ames for providing us with a copy of the Navier-Stokes Code ARC2D. 


\section{References}

1. T. H. Pulliam and D. S. Chaussee, J. Comput. Phys. 39, 347 (1981).

2. F. T. Smith and J. H. Merkin, J. Comput. Phys. 10, 7 (1982).

3. A. E. P. Veldman, AIAA J. 19, 79 (1981).

4. R. T. Davis, AIAA Paper No. 84-1614 (1984).

5. A. H. Nayfeh, S. A. Ragab, and A. Al-Maaitah, Phys. Fluids 31, 796 (1988).

6. R. T. Davis and M. J. Werle, in Numerical and Physical Aspects of Aerodynamics Flows, edited by T. Cebeci (Springer, Berlin 1982), p. 187.

7. R. R. Scott and H. A. Watts, SIAM J. Num. Anal. 14, 40 (1977).

8. R. C. Krishna, MS Thesis, Virginia Polytechnic Institute and State University, Blacksburg, Virginia (1988). 


\section{Figure Captions}

Figure 1. A typical computational grid.

Figure 2. Influence of grids on the skin-friction coefficient for $M_{\infty}=0.5$ and $\operatorname{Re}$ $=1.0 \times 10^{6}$ : 1 IBL, - - 2 NS (GRID1), 3 NS (GRID2), and ... 4 NS (GRID3).

Figure 3. Influence of grids on the pressure coefficient for $M_{\infty}=0.5$ and $R e=1.0 \times 10^{6}$ : $1 \mathrm{IBL},-\cdots 2$ NS (GRID1), 3 NS (GRID2), and ... 4 NS (GRID3).

Figure 4. Influence of grids on the growth rates for $M_{\infty}=0.5$ at $R e=1.0 \times 10^{6}$ and $F=80 \times 10^{-6}$ : 1 IBL, - - - 2 NS (GRID1), 3 NS (GRID2), and ... 4 NS (GRID3).

Figure 5. Influence of grids on the $\mathrm{N}$-factor for $M_{\infty}=0.5$ at $R e=1.0 \times 10^{6}$ and $\mathrm{F}$ $=80 \times 10^{-3}$ : 1 IBL, - - 2 NS (GRID1), 3 NS (GRID2), and ... 4 NS (GRID3).

Figure 6. Comparison of the skin-friction coefficient calculated by using interacting boundary layers with those calculated by using the Navier-Stokes solver and different grid refinements for $M_{\infty}=0.5$ and $R e=1.0 \times 10^{6}$ : $1 \mathrm{IBL}$, - - 2 NS (GRID1), 3 NS (GRID2), ... 4 NS (GRID3), and 5 NS (GRID4).

Figure 7. Comparison of the growth rates based on the profile calculated by using the interacting boundary layer code with those based on the 
profile calculated by using the Navier-Stokes solver with the finest grid for $M_{\infty}=0.5, \operatorname{Re}=1.0 \times 10^{6}$, and $F=80 \times 10^{-6}$ : $1 \mathrm{IBL}$ and --2 NS (GRID4).

Figure 8. Comparison of the $\mathrm{N}$-factors based on the profile calculated by using the interacting boundary layer code with those based on the profiles calculated by using the Navier-Stokes solver with different grid refinements for $M_{\infty}=0.5, \operatorname{Re}=1.0 \times 10^{6}$, and $F=80 \times 10^{-6}$ : $1 \mathrm{IBL}$, - - 2 NS (GRID1), 3 NS (GRID2), .. 4 NS (GRID3), and 5 NS (GRID4).

Figure 9. Comparison of the growth rates based on the profile calculated by using the interacting boundary layer code with those based on the profile calculated by using the Navier-Stokes solver with the finest grid for $M_{\infty}=0.8, \operatorname{Re}=1.0 \times 10^{6}$, and $F=50 \times 10^{-6}$ : $I B L$, and -- NS (GRID4).

Figure 10. Comparison of the $\mathrm{N}$-factors based on the profile calculated by using the interacting boundary layer code with those based on the profile calculated by using the Navier-Stokes solver with the finest grid for $M_{\infty}=0.8, R e=1.0 \times 10^{6}$, and $F=50 \times 10^{-6}$ : IBL and -- NS (GRID4).

Figure 11. Influence of Mach number on the pressure coefficient at $\mathrm{Re}=$ $1.0 \times 10^{6}:$ $M_{\infty}=0.0, \cdots M_{\infty}=0.5$, and $-M_{\infty}=0.8$.

Figure 12. Influence of Mach number on the skin-friction coefficient at $\mathrm{Re}=$ $1.0 \times 10^{6}:$ $M_{\infty}=0.0, \cdots M_{\infty}=0.5$, and $M_{\infty}=0.8$. 
Figure 13. Influence of Mach number and most dangerous frequency on the growth rates for $\operatorname{Re}=1.0 \times 10^{6}$ : $-M_{\infty}=0.0$ and $F=60 \times 10^{-6}$; - $M_{\infty}=0.5$ and $F=55 \times 10^{-6}$; and $-M_{\infty}=0.8$ and $F$ $=50 \times 10^{-6}$.

Figure 14. Influence of Mach number and most dangerous frequency on the $\mathrm{N}$-factors for $\operatorname{Re}=1.0 \times 10^{6}: \quad M_{\infty}=0.0$ and $\mathrm{F}=60 \times 10^{-6}$; $M_{\infty}=0.5$ and $F=55 \times 10^{-6}$; and $\ldots M_{\infty}=0.8$ and $F=50 \times 10^{-6}$. 


\section{ORIGINAL PAGE IS}

\section{OF POOR QUALITY}

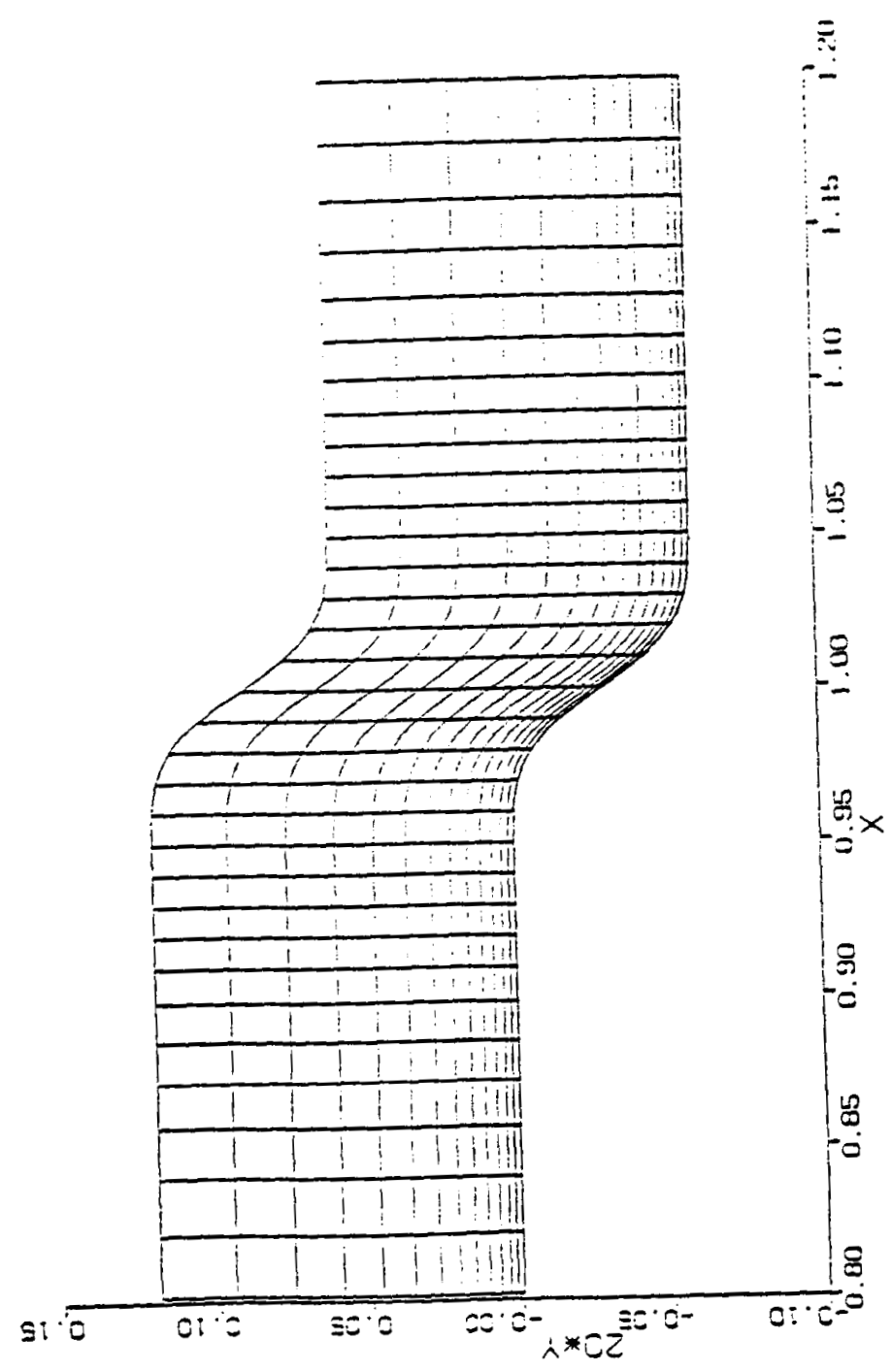




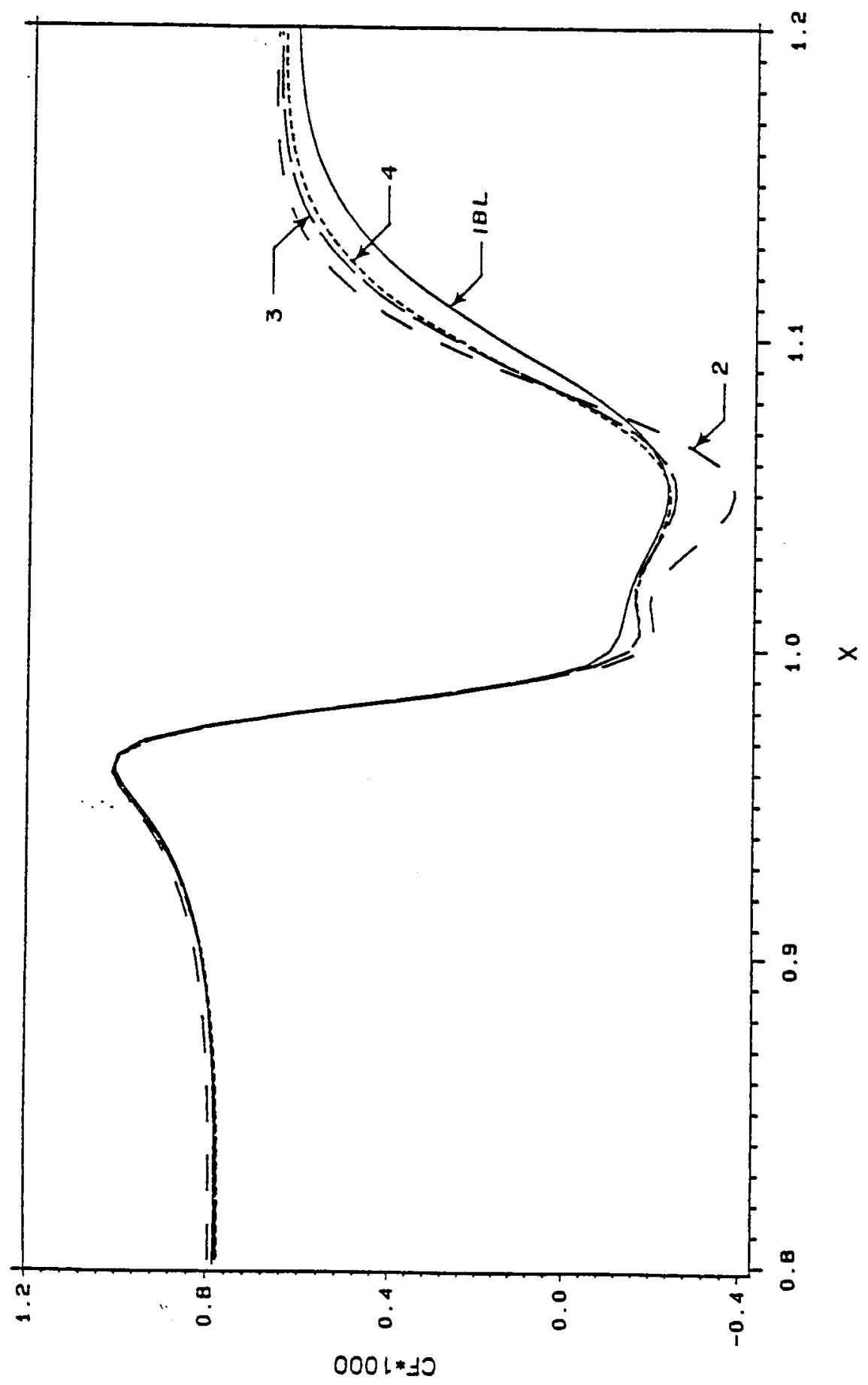




\section{ORIGINAL PAGE IS \\ OF POOR QUALITY}
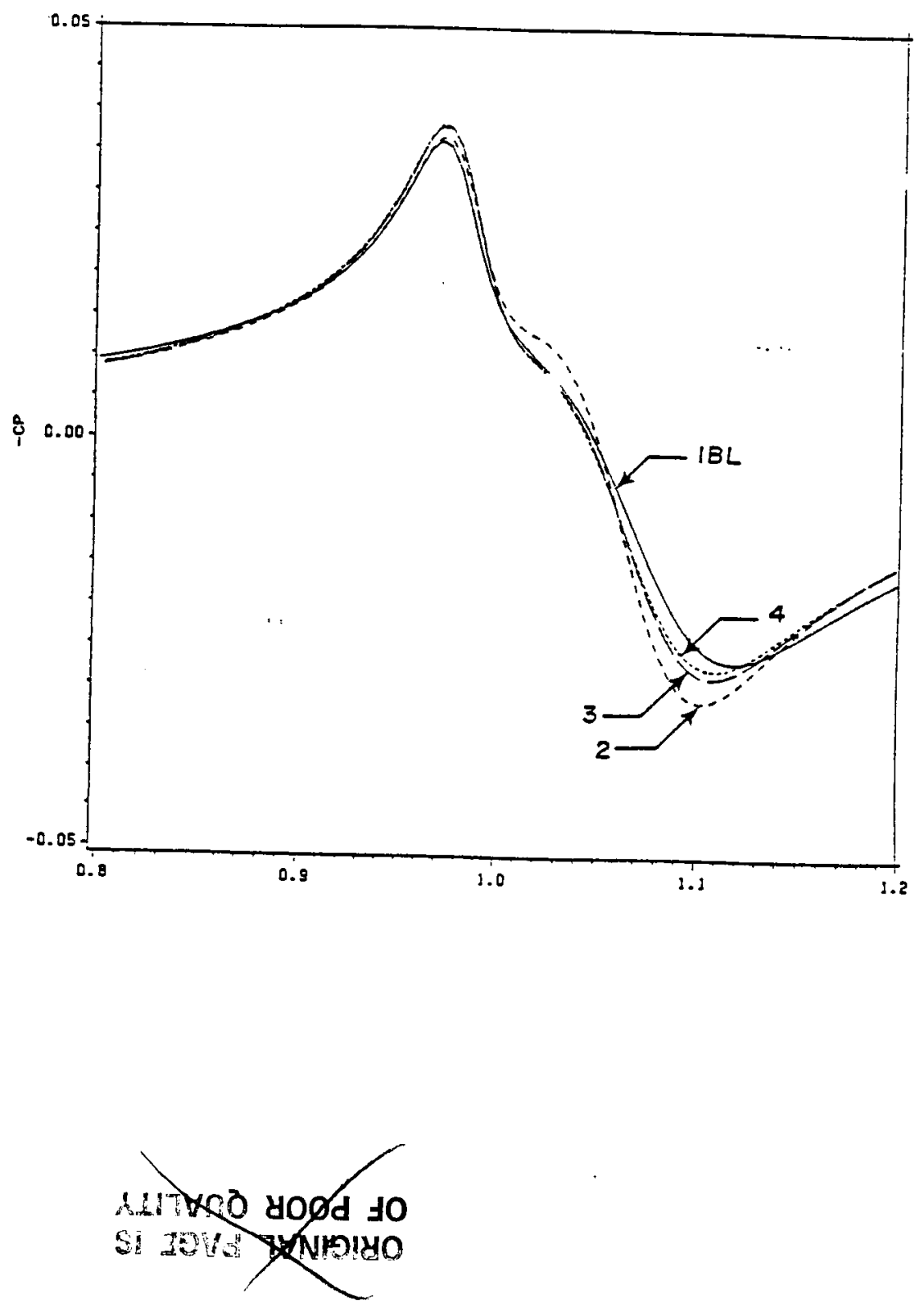
ORIGNAL PAGE IS

OF POOR QUALITY

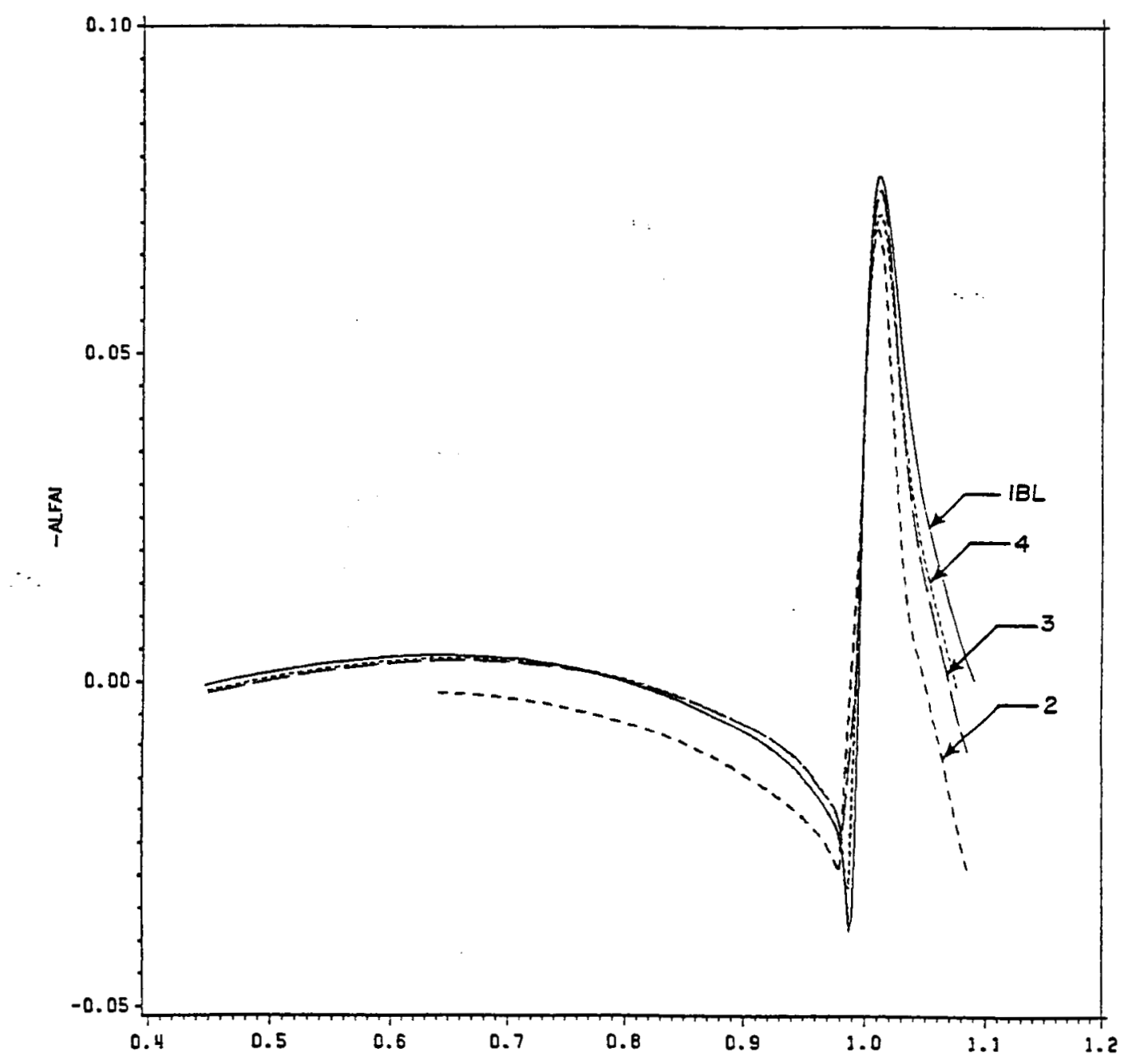


ORIGINAL PAGE IS

OF POOR QUALITY

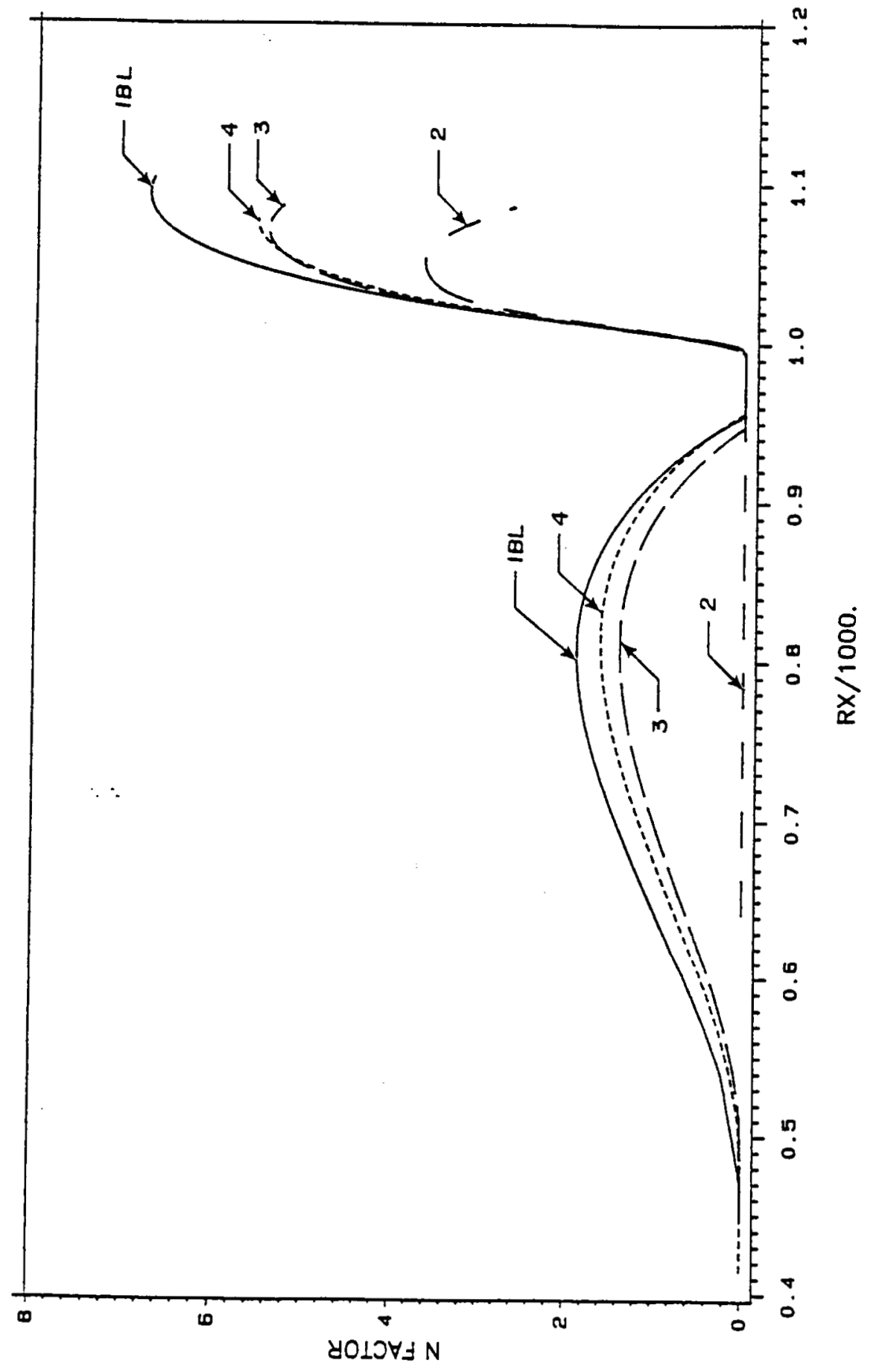


ORIGINAL PAGE IS

OF POOR QUALITY

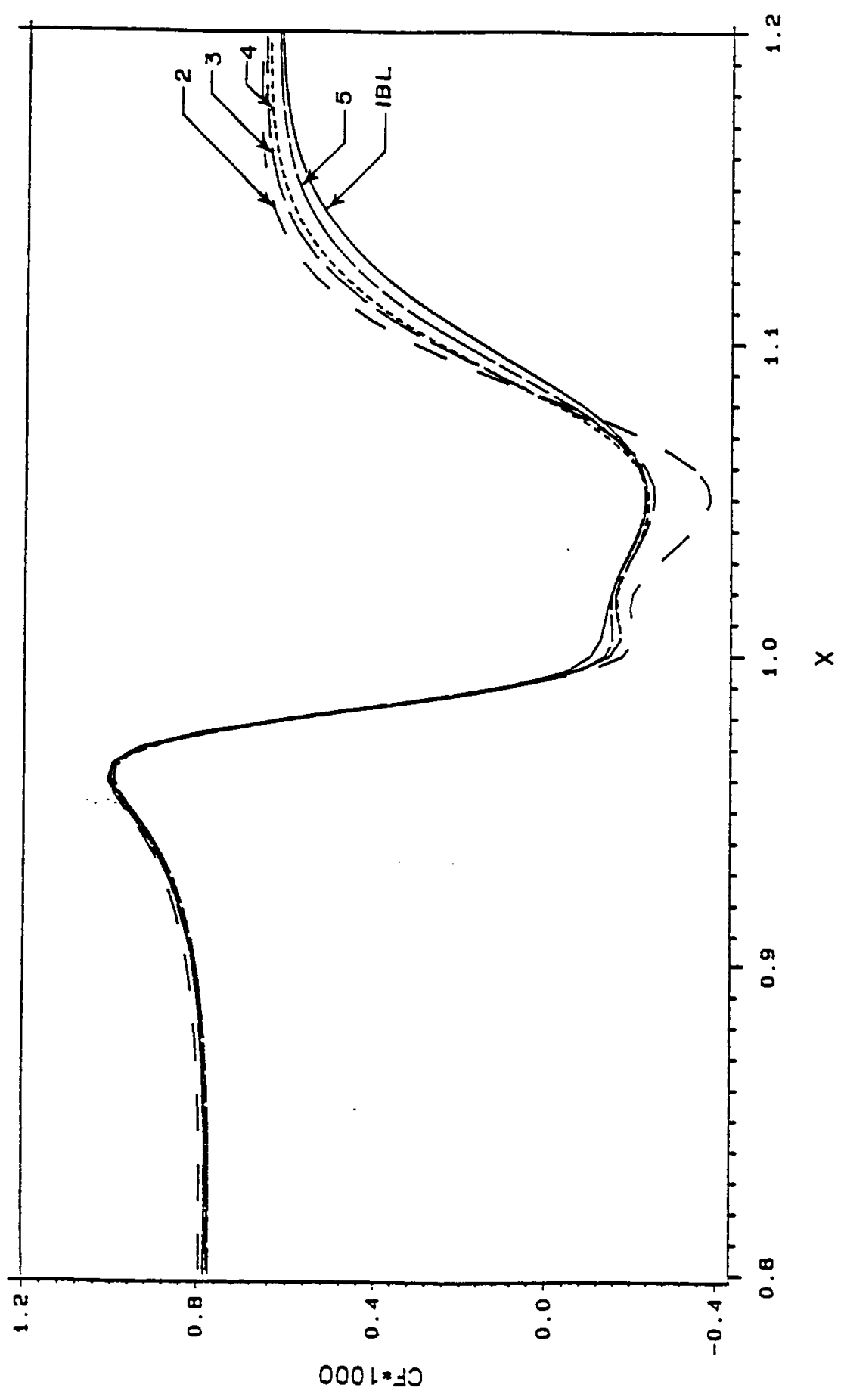




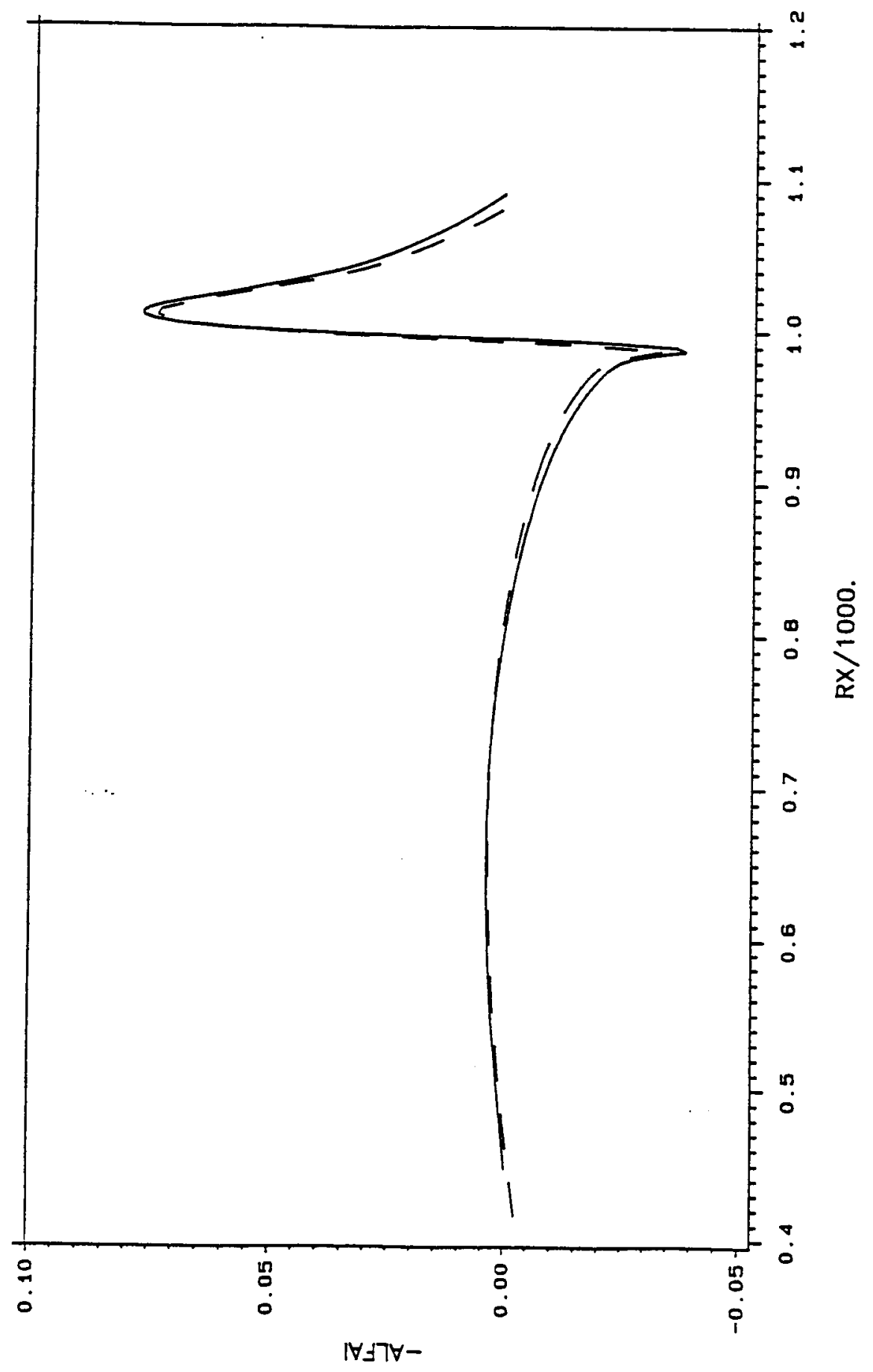




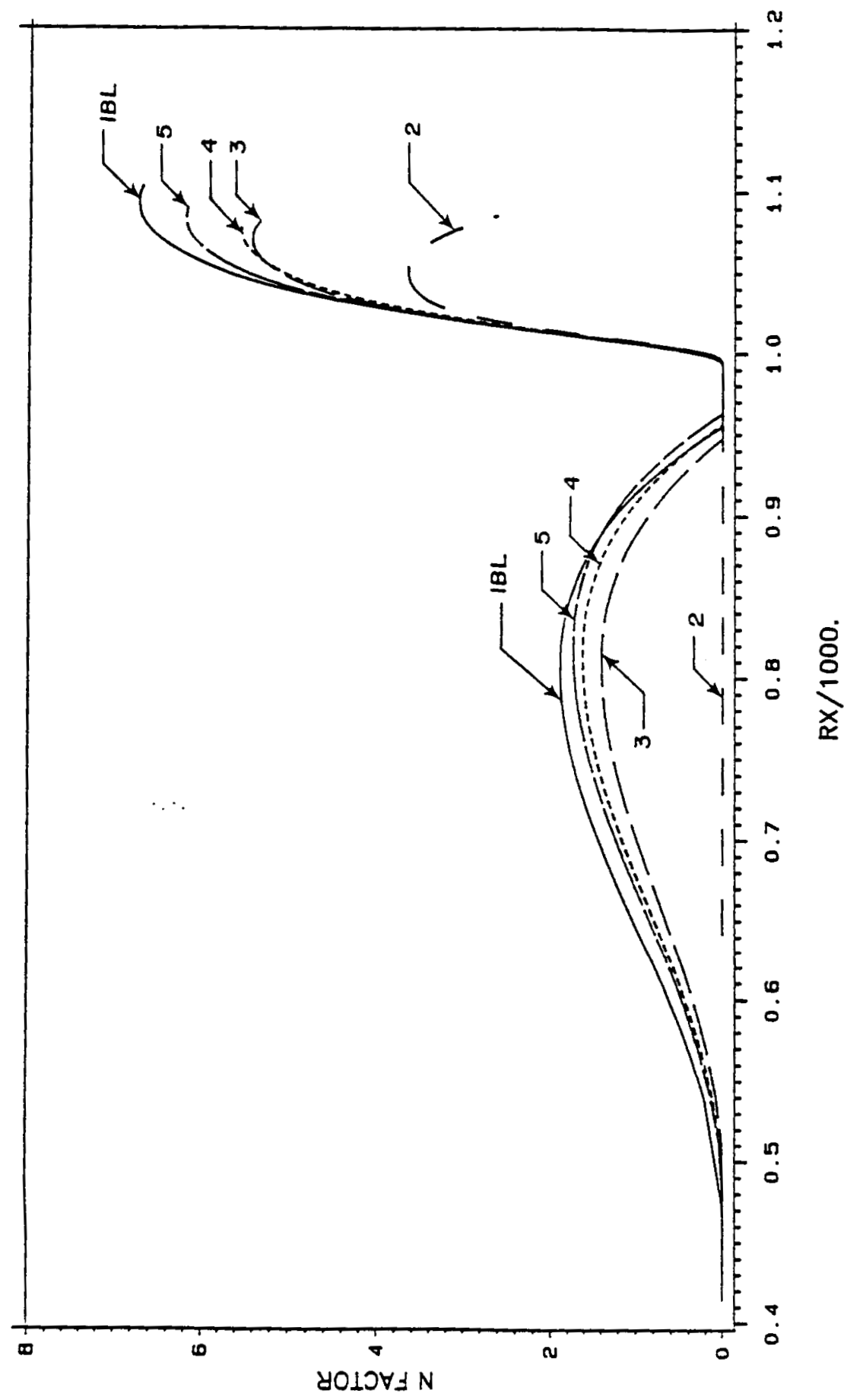




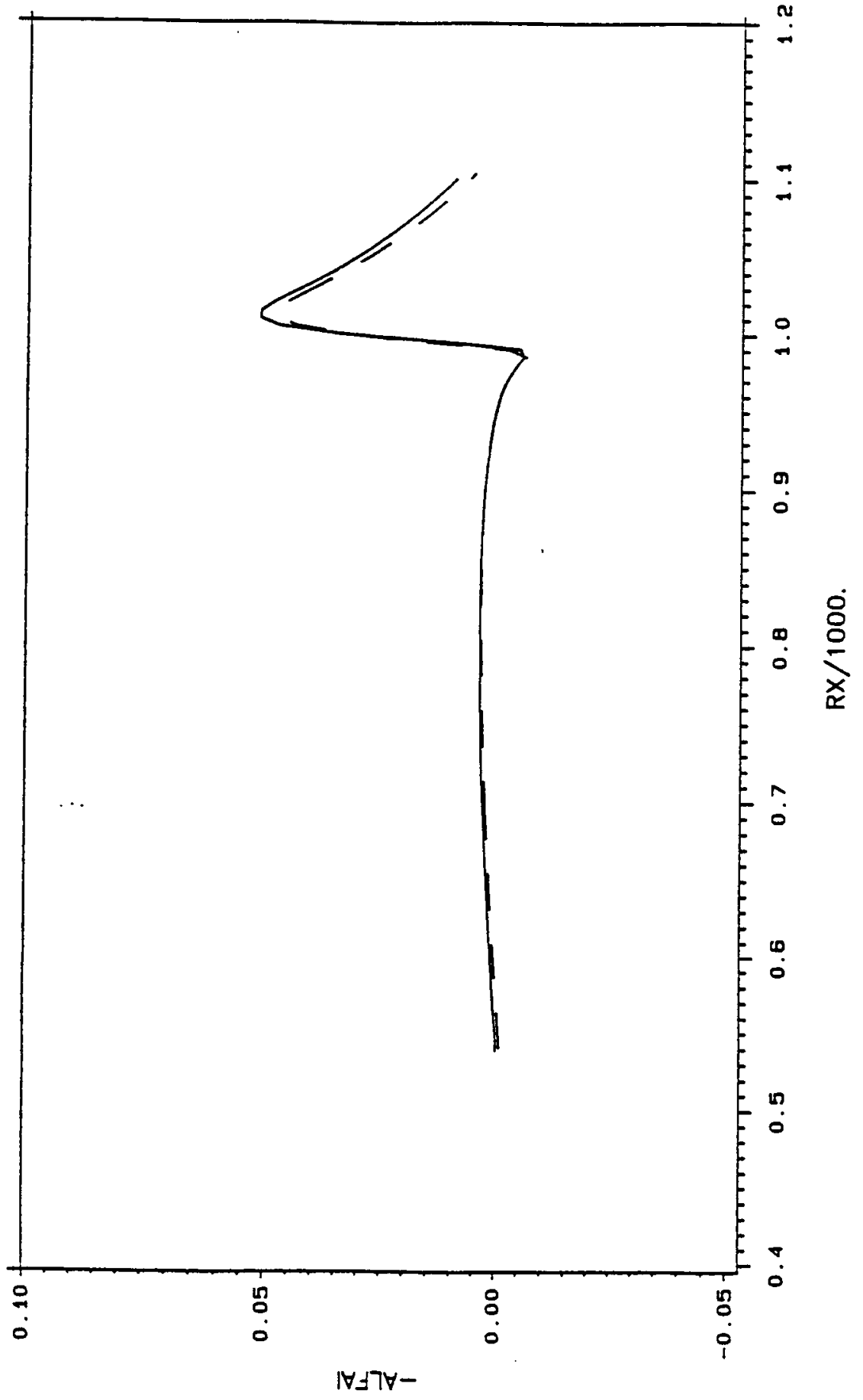




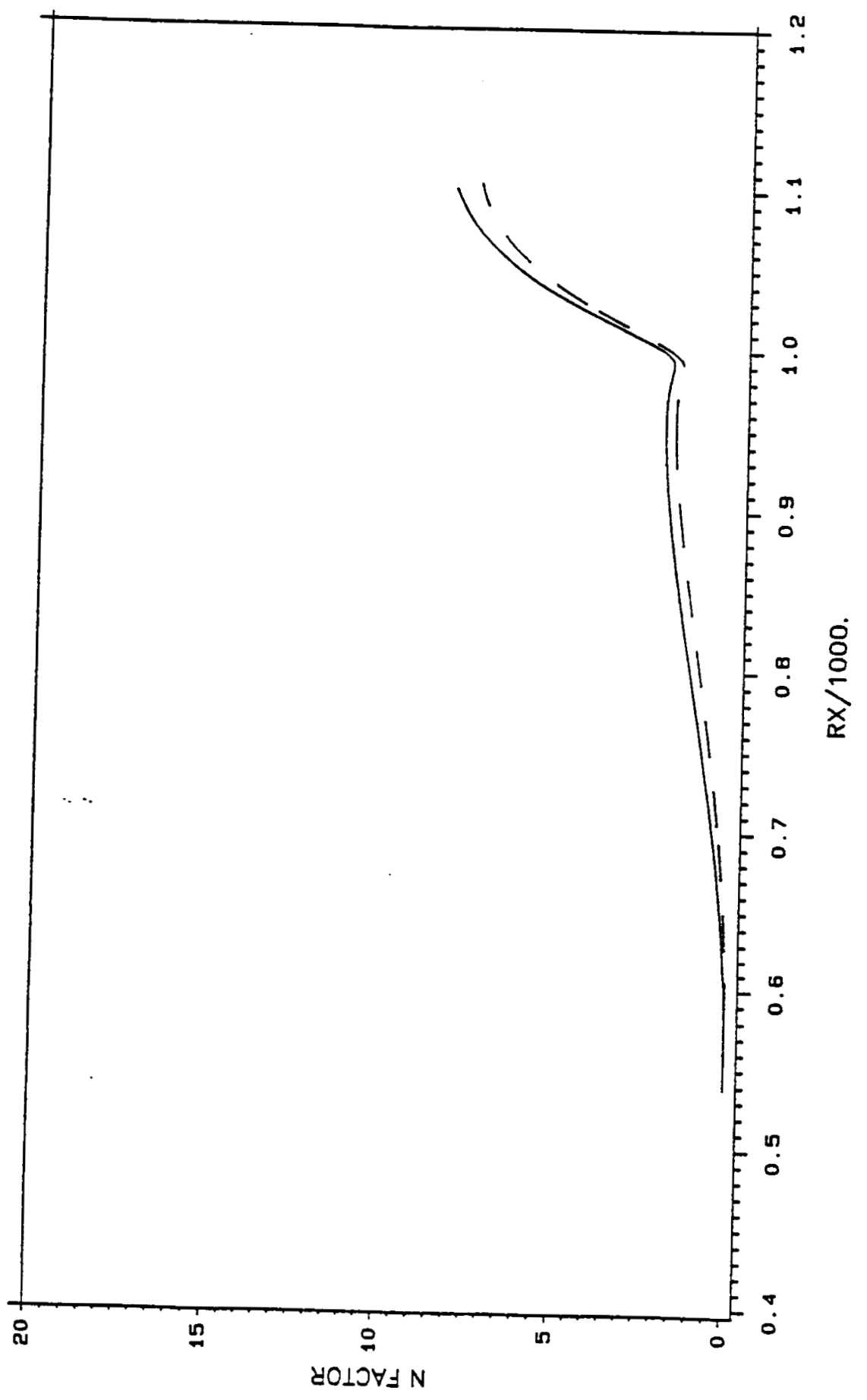




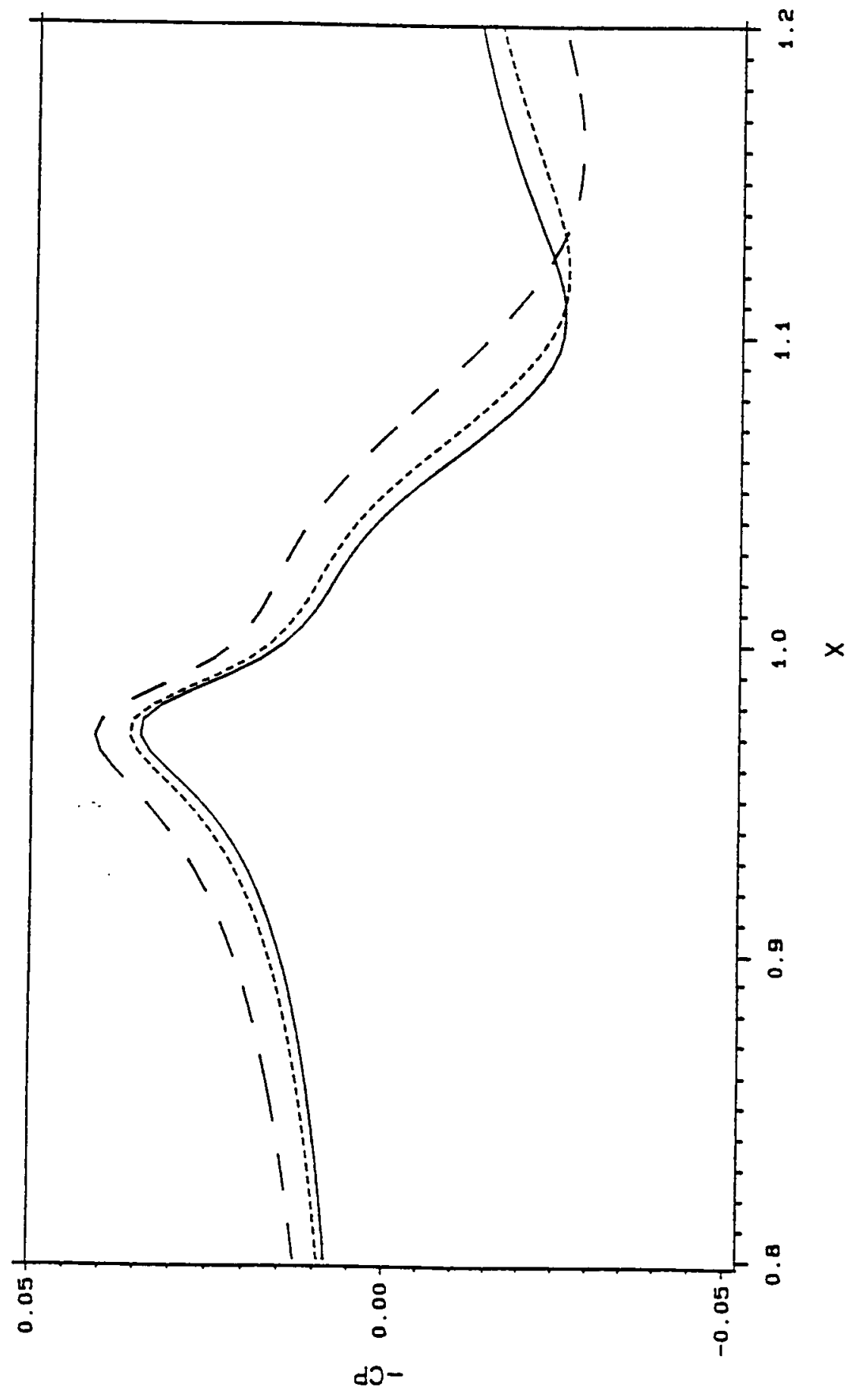




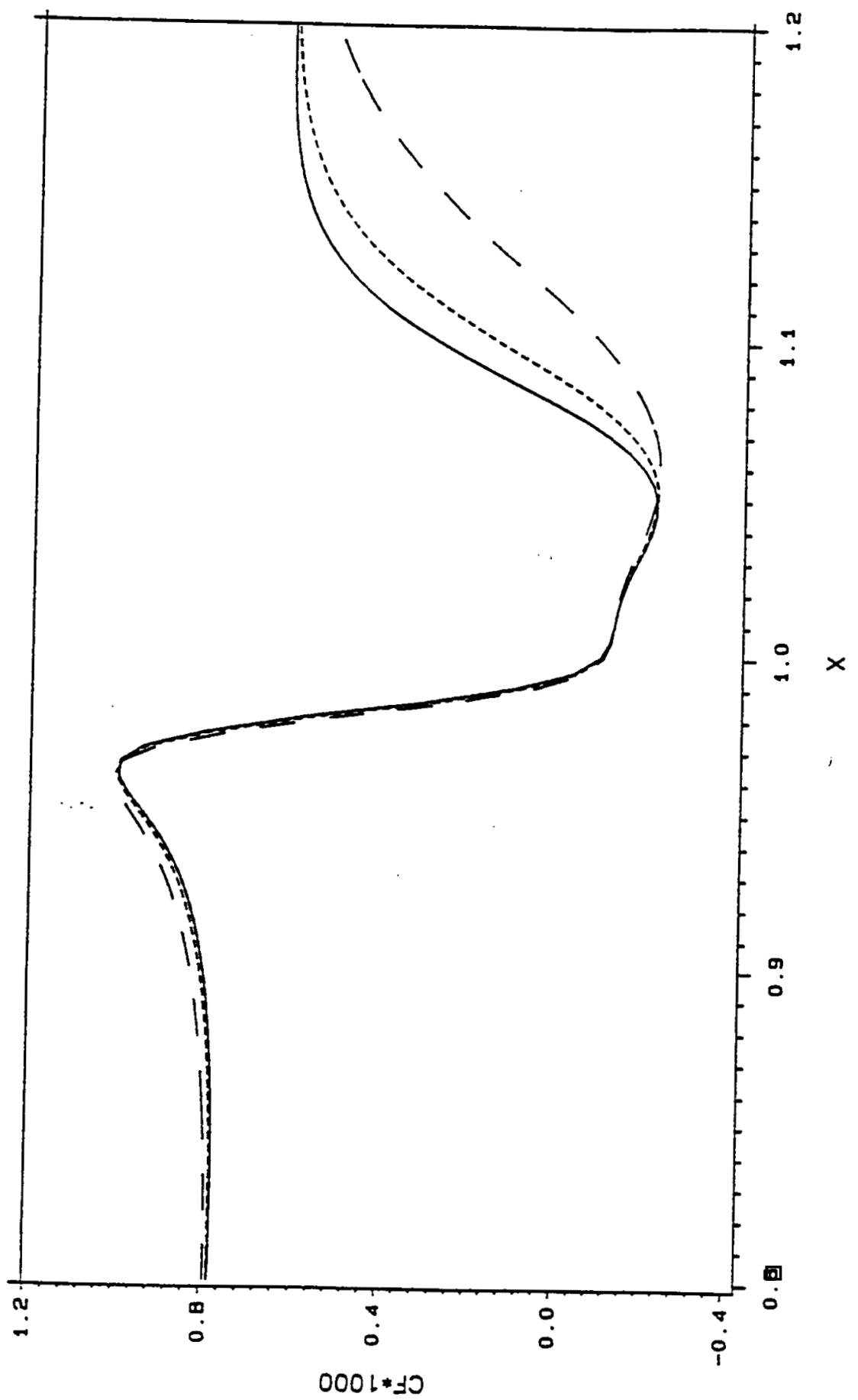




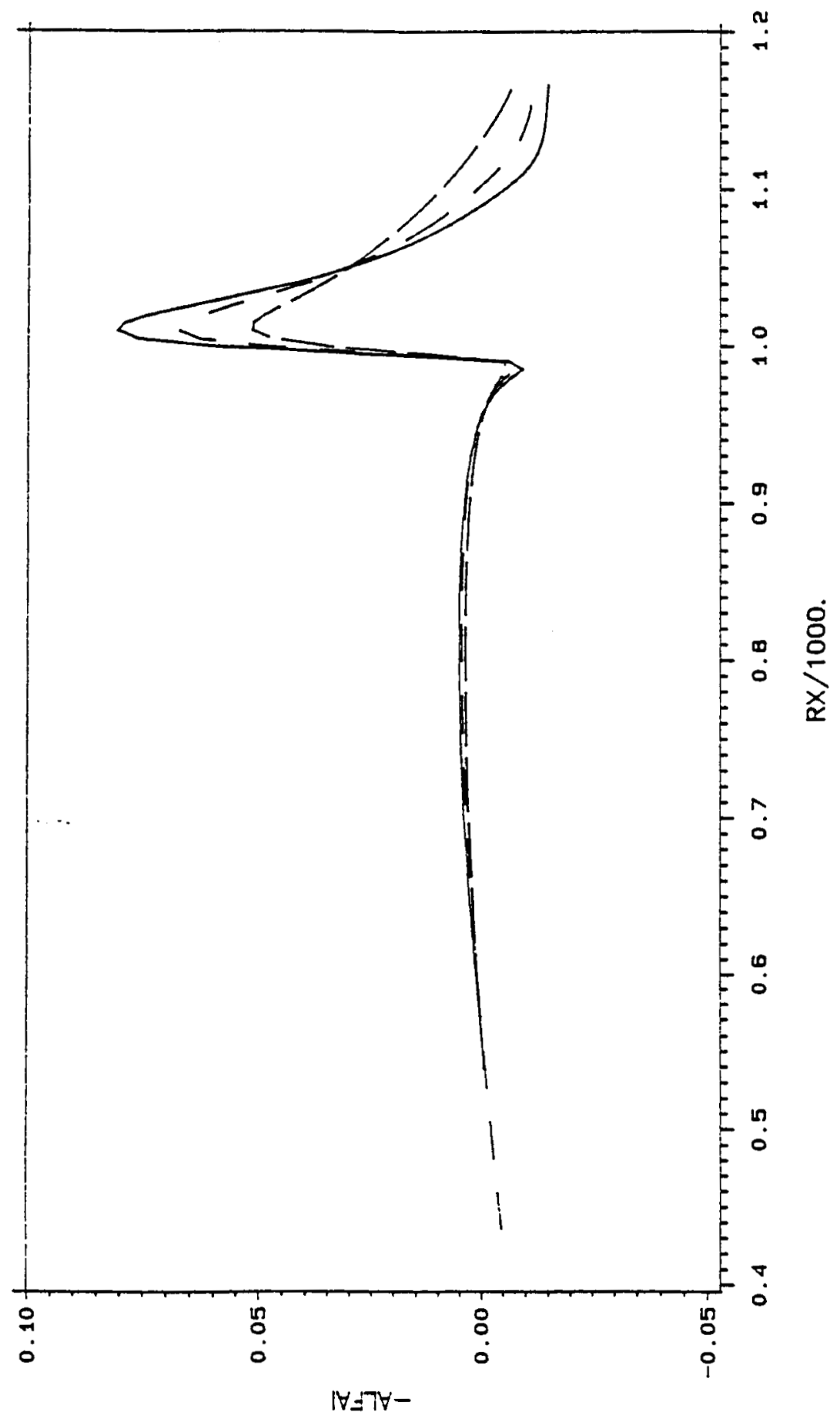




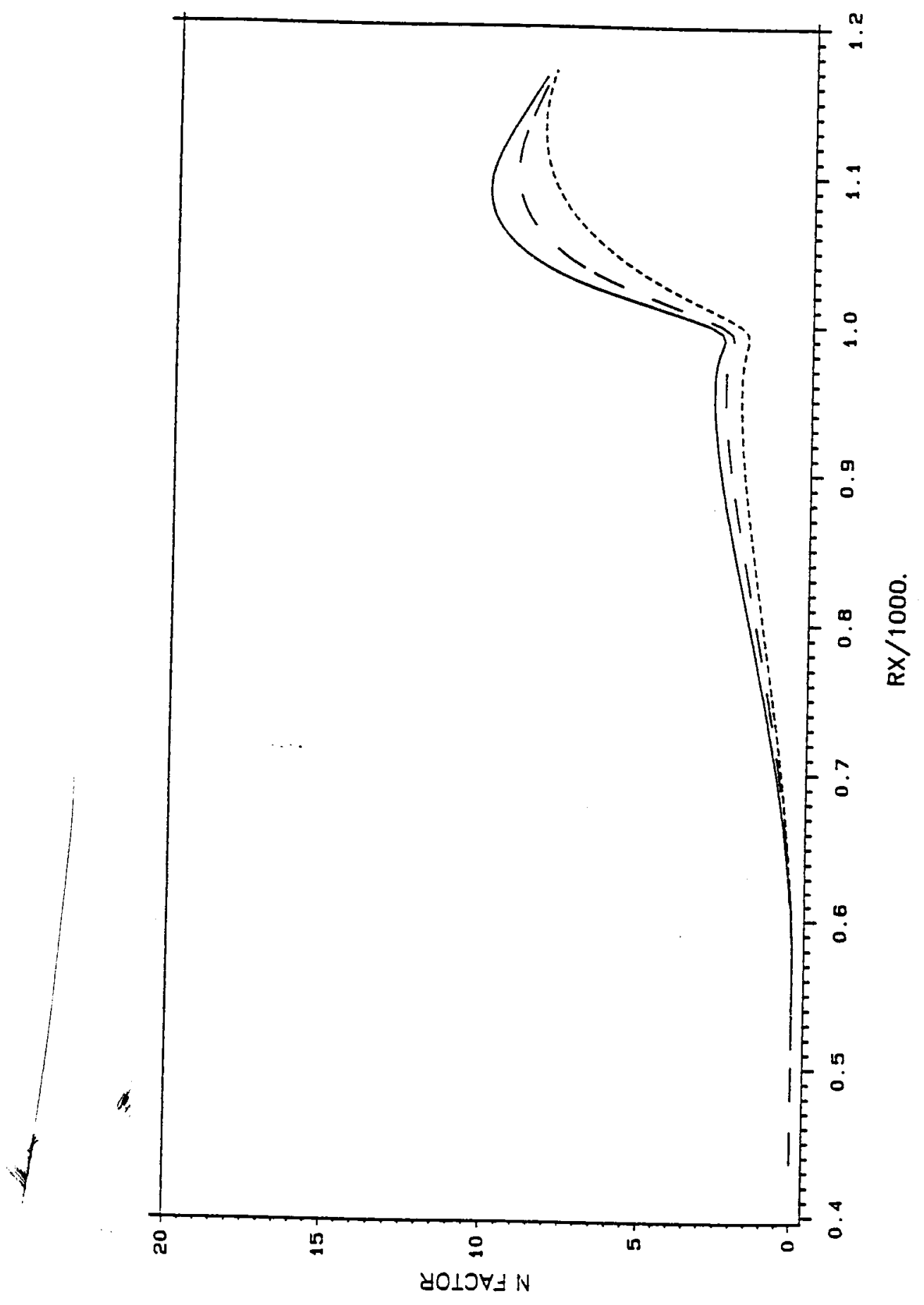

